\title{
Partial response to chemotherapy in a patient with retroperitoneal inflammatory myofibroblastic tumor
}

\author{
MING CHEN ${ }^{1}$, LIHUA ZHANG $^{2}$, GUOCHUN CAO $^{3}$, WEIDONG ZHU $^{1}$, XUQIU CHEN $^{1}$ and QUAN FANG ${ }^{1}$ \\ Departments of ${ }^{1}$ Urology and ${ }^{2}$ Pathology, Zhongda Hospital, Southeast University College of Medicine; \\ ${ }^{3}$ Department of Internal Medicine, Jiangsu Cancer Hospital, Nanjing Medical University, Nanjing, Jiangsu 210009, P.R. China
}

Received March 22, 2016; Accepted May 26, 2016

DOI: $10.3892 / \operatorname{mco} .2016 .967$

\begin{abstract}
Retroperitoneal inflammatory myofibroblastic tumor (IMT) is a rare lesion of unknown etiology. The management of IMT may be challenging due to its intermediate malignant potential, the possibility of local recurrence after surgical resection and its unique anatomic location, which makes radical resection impossible due to its proximity to vital structures. Thus, treatment for recurrence and metastasis mainly relies on chemotherapy. We herein report a case of a 60-year-old man with an IMT sized $6.7 \times 5.1 \mathrm{~cm}$, located in the left adrenal area, which was identified by a computed tomography (CT) scan of the abdomen. Radical surgical resection of the tumor was not feasible, as it was fixed to the left renal artery. After 5 months, the patient underwent CT-guided radiofrequency ablation of the re-growing tumor. An unresectable mass was detected in the patient's rectum by a CT scan of the pelvis. Subsequently, the patient underwent chemotherapy for the recurring and metastatic tumors. The chemotherapeutic regimens included epirubicin, dacarbazine and docetaxel. Over the last 6 months, after three cycles of therapy, the sizes of the primary and metastatic tumors had decreased on the follow-up CT scan. Thus, chemotherapy effectively controlled the disease in this case, following unsuccessful surgical resection and radiofrequency ablation. The present case report highlights the complexity of treatment in such cases and the significance of designing a clinical protocol for the treatment of IMT.
\end{abstract}

\section{Introduction}

Inflammatory myofibroblastic tumor (IMT) is a distinct neoplasm characterized by spindle cell proliferation and an inflammatory infiltrate (1). IMTs located in the retroperitoneum

Correspondence to: Dr Quan Fang, Department of Urology, Zhongda Hospital, Southeast University College of Medicine, 87 Dingjia Qiao Road, Nanjing, Jiangsu 210009, P.R. China E-mail: quanfang@hotmail.com

Key words: inflammatory myofibroblastic tumor, retroperitoneum, chemotherapy are relative rare (2). The management of this type of tumor may be challenging, as there are currently no established protocols and the tumors are occasionally unresectable due to their large size and proximity to vital structures. We herein present a case of a retroperitoneal IMT metastatic to the rectum, which was effectively controlled by chemotherapy following unsuccessful surgical resection and radiofrequency ablation.

\section{Case report}

The patient was a 60 -year-old male who was admitted to a local hospital due to upper abdominal pain for 5 months. The patient described the pain as continuous and dull, radiating to the left flank, and he reported a weight loss of $12 \mathrm{~kg}$ over the past 5 months. The physical examination was unremarkable. A computed tomography (CT) scan of the abdomen and pelvis revealed a solid mass in the left adrenal area. The mass measured $6.7 \times 5.1 \mathrm{~cm}$ and its CT value was 30 Hounsfield units. The density of the mass was enhanced with intravenous contrast administration (Fig. 1A). Non-retroperitoneal lymph nodes were observed on cross-sectional imaging. A diagnosis of retroperitoneal tumor was hypothesized, but the presence of an adrenal mass could not be excluded. The patient was then referred to our institution and subsequently underwent laparoscopic surgery for the resection of the retroperitoneal mass and the right adrenal gland. Macroscopically, the mass was irregular, firm, measuring $9 \mathrm{~cm}$ in greatest diameter. Histological examination revealed loosely arranged spindle cells with admixed collagen bundles and scattered inflammatory cells (Fig. 2A), mainly comprising lymphocytes and plasma cells (Fig. 2B). The proliferation extended into the adjacent nerves, fatty tissue and adrenal gland. The surgical margin was positive for tumor invasion. The immunohistological examination of the tumor was positive for CD35, CD163, vimentin and Ki67 (10\%), and negative for CD21, CD23, CD34, pancytokeratin, S-100, desmin, smooth muscle antigen and anaplastic lymphoma kinase (ALK)-1. A follow-up CT of the abdomen and pelvis revealed progression of the tumor 2 months after surgical resection. The tumor was sized $2.6 \times 2.3 \mathrm{~cm}$ and was located between the aorta and the left diaphragmatic angle (Fig. 1B). The patient refused further treatment and no action was taken, except for close surveillance. Five months after the surgery, a repeat CT of the abdomen and pelvis revealed that the size of the mass had increased to $5.8 \times 4.3 \times 6.5 \mathrm{~cm}$ (Fig. 3A). 

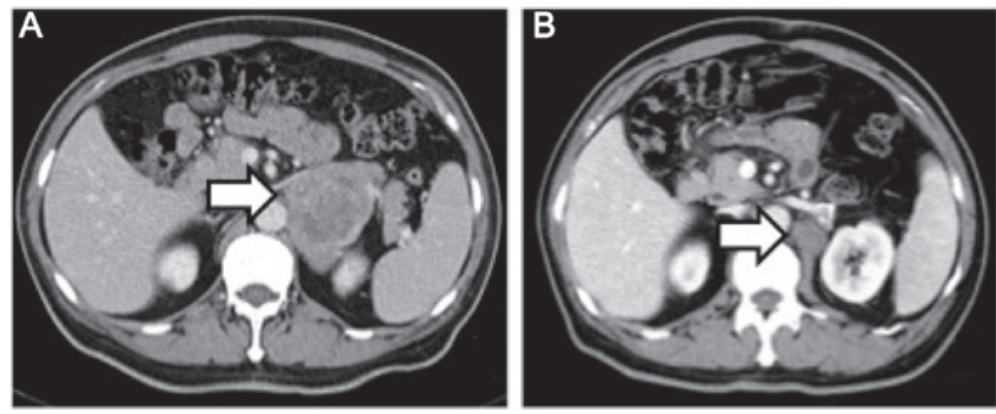

Figure 1. (A) Computed tomography scan of abdomen showing a low-density solid mass with an uneven central cystic lesion in the left adrenal region (arrow); (B) a smaller mass is seen in the same area following surgical resection (arrow).
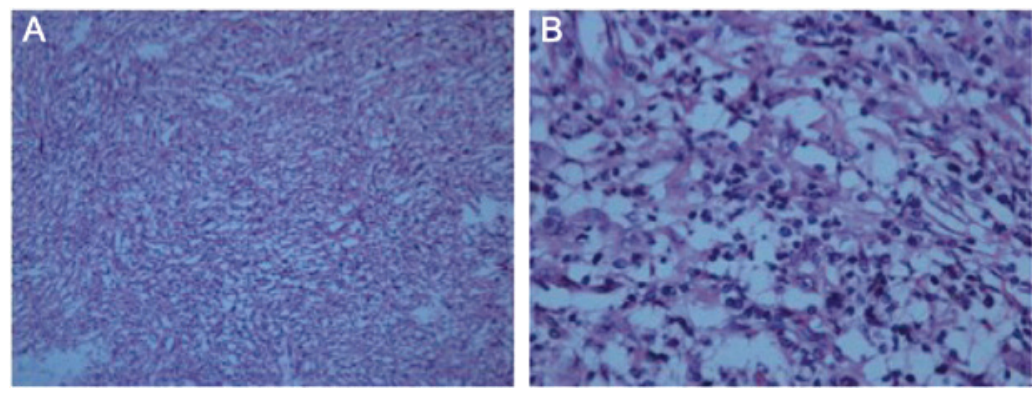

Figure 2. Histological examination by hematoxylin-eosin staining revealed an inflammatory myofibroblastic tumor composed of loosely arranged spindle cells intermixed with collagen bundles and scattered lymphocytes and plasma cells. Magnification (A) x100 and (B) x400.
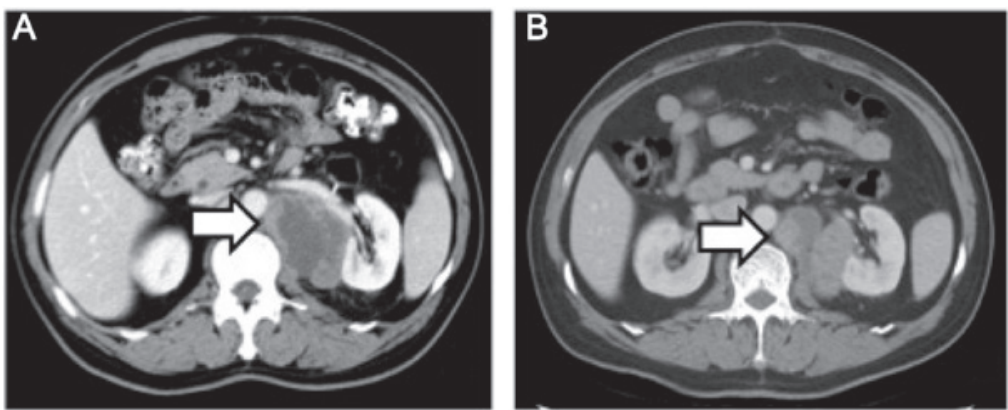

Figure 3. (A) Computed tomography scan showing increased size of the mass compared with Fig. 1B (arrow). (B) The size of the mass decreased following chemotherapy (arrow).
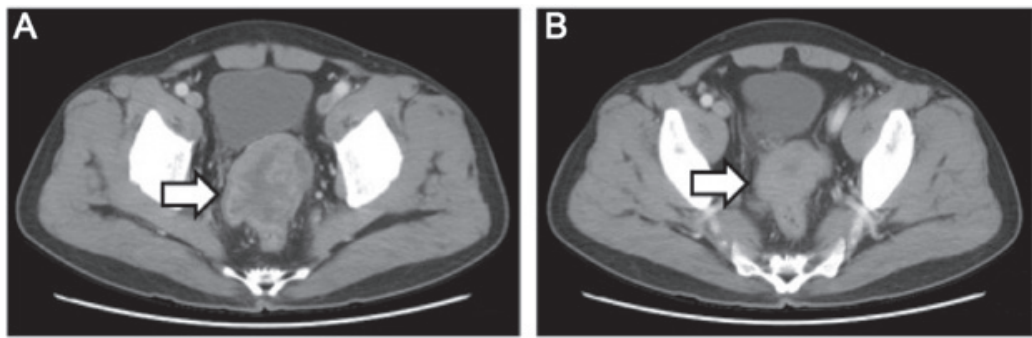

Figure 4. (A) Computed tomography scan of the pelvis showing a large mass located between the bladder and the rectum (arrow). (B) The size of the mass decreased following chemotherapy (arrow).

The patient underwent CT-guided radiofrequency ablation of the retroperitoneal tumor at the Jiangsu Cancer Hospital; however, shortly after the second surgery, an unresectable metastatic tumor was detected in the rectum by CT examination (Fig. 4A). Subsequently, the patient underwent three cycles of chemotherapy for the tumor metastasis. The chemotherapy regimen included epirubicin $50 \mathrm{mg}$ on days 1 and 2; dacarbazine $200 \mathrm{mg}$ daily on days $1-5$; and $50 \mathrm{mg}$ docetaxel on day 1 . The patient tolerated the chemotherapy well. In a recent CT scan (November 15, 2015), the growth of the retroperitoneal 
tumor, as well as that of the metastatic tumor in the rectum, had been stabilized (Figs. 3B and 4B).

All procedures performed were in accordance with the ethical standards of the Institutional Research Committees of the participating institutions and informed consent was obtained from the patient regarding the publication of the case details.

\section{Discussion}

IMT is a neoplasm of intermediate biological potential, which is characterized by spindle cell proliferation with an inflammatory infiltrate. IMT may also be referred to as inflammatory pseudotumor, pseudosarcomatous myofibroblastic proliferation, inflammatory sarcoma, plasma cell granuloma and inflammatory myohistocytic proliferation (3). This disease typically arises in the lung, and rarely in sites such as the retroperitoneum, pelvis, head/neck and extremities $(4,5)$.

The diagnosis of IMT is made on the basis of histological analysis and there are no established decisive criteria for differential diagnosis. Histologically, the lesion is predominantly composed of myofibroblasts in a myxoid to collagenous stroma admixed with inflammatory cells, characterized by paucicellular to moderately cellular spindle- to stellate-shaped cells, with an admixed inflammatory infiltrate of varying density (6). The characteristics of this disease vary between different sites, possibly indicating the different phases of its development. The major characteristics include a spectrum of myofibroblastic proliferation, often with a vaguely storiform architecture, along with varying amounts of inflammatory infiltrates, mainly consisting of lymphocytes, histiocytes and plasma cells. The presence of scattered eosinophils has also been reported in a number of cases, particularly in the head of pancreatic IMTs (2).

Although initially IMT was considered to be a benign reactive lesion, this hypothesis has been challenged due to the neoplastic characteristics in the clinical presentation, with a high recurrence rate of $18-40 \%$, the presence of regional metastasis, and the acquired clonal chromosomal abnormality in the cytogenetic analysis (7). Of all the chromosomal aberrations identified in the disease, the chromosomal region 2 p23 near or within the ALK gene is consistently involved in IMT (8). Rearrangements involving the ALK locus on chromosome 2p23 have been documented in $~ 50 \%$ of IMTs (6). Distant metastases occur primarily in ALK-negative IMTs, but local recurrence occurs regardless of ALK expression (4). In the present case, the specimen stained negative for ALK and rectal metastasis occurred shortly after surgical removal of the primary tumor.

The management of IMTs may be challenging, as there are currently no established treatment protocols; radical local excision of the tumor is the mainstay of treatment. IMTs occurring in the abdomen or retroperitoneum often have a propensity for more aggressive behavior with multiple recurrences, invasion into adjacent structures and metastases, making curative resection impossible and promoting local recurrence (4). In our patient, the first attempt of radical excision of the tumor was unsuccessful due to its proximity to the left renal artery, resulting in a positive margin, which led to tumor recurrence. Adjuvant therapy would be indicated in this case; however, a standardized guideline for chemotherapy and radiotherapy is not available, due to the rarity of the disease. Although the progression of the primary mass was controlled by CT-guided radiofrequency ablation, a distant metastasis to the rectum made further management necessary; chemotherapy was the treatment of choice for this patient.

In the published literature, multiple agents have been investigated in the treatment of malignant IMTs, including vincristine, etoposide, cisplatin, adriamycin and methotrexate (9-11). Non-steroidal anti-inflammatory drugs have also been added to the regimens, with or without chemotherapeutic agents (12). However, the clinical outcomes are difficult to interpret due to the heterogeneity of the treatment modalities. Although novel therapies have been reported for the treatment of the aggressive form of IMT, including anti-inflammatory agents, antitumor necrosis factor- $\alpha$-binding antibodies (13) and, more recently, the ATP-competitive inhibitors of ALK, crizotinib, ceritinib and alectinib $(14,15)$, further application of these agents in clinical practice relies on future clinical trials. Based on extrapolation from studies on soft tissue malignancies and the National Comprehensive Cancer Network recommendations (16), an aggressive regimen was applied in the present case, using a combination of epirubicin, dacarbazine and docetaxel, for the management of the retroperitoneal IMT. After three cycles of chemotherapy, the result is considered encouraging and the growth of the tumor appears to have been arrested.

To the best of our knowledge, this is the only published case report on the management of an aggressive retroperitoneal IMT with epirubicin, dacarbazine and docetaxel combination chemotherapy achieving a partial response in the primary as well as the metastatic site. Although the results are encouraging, the long-term prognosis of this patient is uncertain, since the tumor has not been completely eliminated. Although a third surgery attempting complete resection of the tumor may be an option, given the nature of this disease, the long-term survival prediction is not favorable due to the propensity of the lesion for invasion and metastasis. Moreover, a laparotomy after chemotherapy would be challenging and decision making regarding surgery depends on the skill and experience of the surgical team, as well as on the patient's overall condition. In the future, thorough investigation of the heterogeneous nature of this disease and subsequent development of targeted therapies may result in the successful management of malignant IMTs.

\section{References}

1. Coffin CM, Dehner LP and Meis-Kindblom JM: Inflammatory myofibroblastic tumor, inflammatory fibrosarcoma and related lesions: An historical review with differential diagnostic considerations. Semin Diagn Pathol 15: 102-110, 1998.

2. Pungpapong S, Geiger XJ and Raimondo M: Inflammatory myofibroblastic tumor presenting as a pancreatic mass: A case report and review of the literature. JOP 5: 360-367, 2004.

3. Yamamoto H, Watanabe K, Nagata M, Tasaki K, Honda I, Watanabe S, Soda $\mathrm{H}$ and Takenouti T: Inflammatory myofibroblastic tumor (IMT) of the pancreas. J Hepatobiliary Pancreat Surg 9: 116-119, 2002.

4. Coffin CM,Watterson J, Priest JR and Dehner LP: Extrapulmonary inflammatory myofibroblastic tumor (inflammatory pseudotumor). A clinicopathologic and immunohistochemical study of 84 cases. Am J Surg Pathol 19: 859-872, 1995.

5. Ziadi S, Trimeche M, Mestiri S, Boujelbene N, Mokni M, Sriha B and Korbi S: Retroperitoneal myofibroblastic inflammatory tumor. Tunis Med 89: 400-401, 2011. 
6. Coffin CM, Hornick JL and Fletcher CD: Inflammatory myofibroblastic tumor: Comparison of clinicopathologic, histologic, and immunohistochemical features including ALK expression in atypical and aggressive cases. Am J Surg Pathol 31: 509-520, 2007.

7. Bertocchini A, Lo Zupone C, Callea F, Gennari F, Serra A, Monti L and de Ville de Goyet J: Unresectable multifocal omental and peritoneal inflammatory myofibroblastic tumor in a child: Revisiting the role of adjuvant therapy. J Pediatr Surg 46 : e17-e21, 2011.

8. Griffin CA, Hawkins AL, Dvorak C, Henkle C, Ellingham T and Perlman EJ: Recurrent involvement of 2p23 in inflammatory myofibroblastic tumors. Cancer Res 59: 2776-2780, 1999.

9. Dishop MK, Warner BW, Dehner LP, Kriss VM, Greenwood MF Geil JD and Moscow JA: Successful treatment of inflammatory myofibroblastic tumor with malignant transformation by surgical resection and chemotherapy. J Pediatr Hematol Oncol 25 $153-158,2003$

10. Firat $\mathrm{O}$, Ozturk $\mathrm{S}$, Akalin $\mathrm{T}$ and Coker A: Inflammatory myofibroblastic tumour. Can J Surg 52: E60-E61, 2009.

11. Tao YL, Wang ZJ, Han JG and Wei P: Inflammatory myofibroblastic tumor successfully treated with chemotherapy and nonsteroidals: A case report. World J Gastroenterol 18 : 7100-7103, 2012
12. Przkora R, Bolder U, Schwarz S, Jauch KW, Spes J, Andreesen R and Mackensen A: Regression of nonresectable inflammatory myofibroblastic tumours after treatment with nonsteroidal anti-inflammatory drugs. Eur J Clin Invest 34: 320-321, 2004.

13. Berger A, Kim C, Hagstrom N and Ferrer F: Successfu preoperative treatment of pediatric bladder inflammatory myofibroblastic tumor with anti-inflammatory therapy. Urology 70 : 372.e13-e15, 2007.

14. Katayama R, Lovly CM and Shaw AT: Therapeutic targeting of anaplastic lymphoma kinase in lung cancer: A paradigm for precision cancer medicine. Clin Cancer Res 21: 2227-2235, 2015.

15. Butrynski JE, D'Adamo DR, Hornick JL, Dal Cin P, Antonescu CR, Jhanwar SC, Ladanyi M, Capelletti M, Rodig SJ, Ramaiya N, et al: Crizotinib in ALK-rearranged inflammatory myofibroblastic tumor. N Engl J Med 363: 1727-1733, 2010.

16. von Mehren M, Randall RL, Benjamin RS, Boles S, Bui MM, Casper ES, Conrad EU III, DeLaney TF, Ganjoo KN, George S, et al: Gastrointestinal stromal tumors, version 2.2014. J Natl Compr Canc Netw 12: 853-862, 2014. 